\title{
TENDÊNCIAS TEMÁTICAS DOS GRUPOS DE PESQUISA EM EDUCAÇÃO EM ENFERMAGEM DO ESTADO DE SÃO PAULO
}

\section{Trends themes of research groups in nursing education in the state of São Paulo}

Bruna Pedroso Canever ${ }^{1}$, Marta Lenise do Prado², Vânia Marli Schubert Backes ${ }^{3}$, Diana Coelho Gomes ${ }^{4}$, Taize Lebarbenchon Briguente ${ }^{5}$, Fabiane Ferraz ${ }^{6}$

${ }^{1}$ Doutora em Enfermagem. Supervisora Técnica na Associação Saúde da Família. São Paulo, Brasil.

${ }^{2}$ Doutora em Enfermagem. Docente do Programa de Pós-Graduação em Enfermagem da Universidade Federal de Santa Catarina (PEN/UFSC). Pesquisadora do CNPq. Florianópolis, Santa Catarina, Brasil.

${ }^{3}$ Doutora em Enfermagem. Docente do Departamento de Enfermagem e do PEN/UFSC. Pesquisadora do CNPq. Florianópolis, Santa Catarina, Brasil.

${ }^{4}$ Mestre em Enfermagem. Helsefagarbeider, Rokilde Sykehjem in Bergan Sykehjem (Profissional municipal da área da saúde, atua em clínicas geriátricas e de tratamento paliativo), Kristiansund Kommune, Vest-Agder, Noruega.

${ }^{5}$ Enfermeira egressa da Universidade do Extremo Sul Catarinense. Criciúma, Santa Catarina, Brasil.

${ }^{6}$ Doutora em Enfermagem. Docente do Curso de Enfermagem e do Programa de PósGraduação em Saúde Coletiva, da Universidade do Extremo Sul Catarinense (UNESC).

\section{Endereço para Correspondência:}

Bruna Pedroso Canever

Rua Maria da Anunciação de Oliveira, 54/Ap. 06

Rio Pequeno, São Paulo, SP, Brasil

CEP: 053390-50

Email: brunacanever@gmail.com 


\title{
Resumo
}

Objetivo: identificar as tendências temáticas e a tradução/aplicação do conhecimento expressos na produção científica dos Grupos de Pesquisa em Educação em Enfermagem (GPEE) do estado de São Paulo/Brasil, destacando as fortalezas e os vazios dessa produção. Método: pesquisa do tipo exploratório-descritiva, em base documental, de natureza quantitativa. Resultados: o total de artigos científicos publicados, no período de 2004 a 2010 com Qualis/CAPES-Enfermagem A1, A2, B1 e B2 foram de 557 estudos. A maior concentração de publicação (268) ocorreu em periódicos, classificados como A2. Houve predomínio de pesquisas qualitativas (265), nas quais os referenciais teóricos mais utilizados foram: fenomenologia e representações sociais. Conclusões: as tendências temáticas Cuidado em Enfermagem e Saúde (146) e Educação, Enfermagem e Saúde (131), tiveram destaque dentro da produção científica, o que revela um fator positivo na produção de artigos científicos dos GPEE, contribuindo com o fortalecimento e avanço da área.

Palavras-chave: Publicações Educação em Enfermagem; Grupos de Pesquisa, Enfermagem.

\begin{abstract}
Objective: to identify thematic trends and the translation/application of the knowledge described in scientific production of Research Groups in Nursing Education (GPEE) of the state of Sao Paulo/Brazil; highlighting the strengths and gaps of this production. Methods: exploratory and descriptive research, based on documentary, and of quantitative nature. Results: the total number of scientific articles published from 2004 to 2010 Qualis/CAPES-Nursing A1, A2, B1 and B2 were 557 studies. The highest concentration of publishing (268) occurred in journals classified as A2. A predominance of qualitative research (265), in which the theoretical reference most frequently used were: phenomenology and social representations. Conclusions: the thematic trends, Nursing and Health Care (146) and Education, Nursing and Health (131) stood out within the production science, which shows a positive factor in the production of scientific articles of Research Groups on Nursing Education, contributing with the strengthening and expansion of the area.
\end{abstract}


Keywords: Scientific and Technical Publications; Education in Nursing; Research Groups; Nursing.

\section{INTRODUÇÃO}

Entre as décadas de 1960 e 1970, conferências desenvolvidas pela Organização das Nações Unidas para a Educação, a Ciência e a Cultura (UNESCO) e pela Organização dos Estados Americanos (OEA) incitaram muitos países da região das Américas a estruturar organismos de Ciência \& Tecnologia com a finalidade de fortalecer o avanço da investigação científica em distintas áreas, incluindo a Enfermagem, por meio de projetos e divulgação da produção científica1.

Nesse cenário, destaca-se o processo de produção do conhecimento da Enfermagem no Brasil, o qual está, historicamente, relacionado ao movimento de estruturação dos Cursos de Pós-Graduação Stricto Sensu em Enfermagem e à formação de Grupos de Pesquisa na área desde a década de 70 do século XX. Ambas as estruturas possibilitaram a consolidação da base científica de Enfermagem, a formação de profissionais capacitados para suprir as demandas sociais, bem como a solução de problemas de cunho regional e nacional, mediante o domínio do conhecimento na área em que exercem suas práticas².

O Brasil, em 2012, contabilizava o total de 83 Cursos de Pós-Graduação em Enfermagem, os quais possuíam vínculo com 58 programas, a saber: $20 \mathrm{com}$ mestrado acadêmico, 25 com mestrado e doutorado acadêmico, 11 com mestrado profissional e 2 com doutorado acadêmico. Destaca-se que com estes programas foi possível formar um número expressivo de pesquisadores enfermeiros brasileiros e de outros países Latino-americanos e da África, bem como profissionais de outras áreas titulados em programas de Enfermagem no Brasil, contribuindo de forma significativa com o crescimento da profissão e reconhecimento da área como produtora de ciência e tecnologia ${ }^{3}$.

Aderidas ao processo de produção do conhecimento da Enfermagem brasileira, encontram-se as tendências temáticas. Essas são compreendidas no presente estudo como um conjunto de assuntos similares que possuem, na sua essência, o mesmo objeto de estudo e que permeiam a produção científica. As 
tendências temáticas evidenciam como possíveis temas vêm sendo trabalhados ao longo do tempo em uma determinada área, nesse caso específico a Enfermagem.

A temática de Educação em Enfermagem merece destaque no processo de produção de conhecimento na área da Enfermagem, visto que as pesquisas desenvolvidas nessa vertente direcionam a formulação de políticas públicas, auxiliam na reformulação de currículos integrados para a formação dos futuros profissionais e contribuem com a formação de recursos humanos qualificados no propósito de prestar uma assistência de qualidade à população, fortalecendo e valorizando a área ${ }^{4}$.

Conforme dados do Censo de 2008, do Conselho Nacional de Desenvolvimento Científico e Tecnológico (CNPq), o Brasil possui cadastrados 51 Grupos de Pesquisa em Educação em Enfermagem (GPEE). Estudos concluídos nas Regiões Sul, Norte, Nordeste e Centro-Oeste revelam que a produção científica na maioria dos GPEE não possui como foco central o seu tema de interesse, ou seja, Educação em Enfermagem, fato que dificulta o avanço no setor de Educação em Saúde e enfraquece a linha de pesquisa que deveria ser a principal dentro dos grupos $^{5-6}$.

Compreende-se a produção científica como os produtos concebidos dentro de uma comunidade científica e que possuem comprovações acerca do assunto trabalhado. Para que ocorra o crescimento de uma disciplina enquanto ciência é necessário que haja a produção e disseminação de conhecimentos que promovam o avanço acerca da prática profissional contribuindo com a melhoria da qualidade de vida dos seres humanos. O desafio da Enfermagem como disciplina da área da saúde está na produção, disseminação e garantia do acesso ao conhecimento ${ }^{7}$.

A tradução/aplicação do conhecimento produzido é compreendida como a capacidade de incorporação desse conhecimento à prática disciplinar, sendo a incorporação/reprodução do discurso uma das possíveis formas de verificar essa ação. Assim, nesse estudo, será utilizado o índice de citação de um artigo como um dos indicadores possíveis de sua tradução/aplicação.

Tendo em vista os resultados publicados sobre como se encontra a produção científica dos GPEE em algumas regiões do Brasil4-10, justifica-se a realização do presente estudo, a fim de conhecer o que vem sendo produzido nos Grupos de Pesquisa em Educação em Enfermagem do estado de São Paulo, visto ser um importante polo acadêmico, com considerável impacto na produção científica da área. 
Assim, o estudo tem por objetivo identificar as tendências temáticas e a tradução/aplicação do conhecimento expressos da produção científica dos Grupos de Pesquisa em Educação em Enfermagem do estado de São Paulo/Brasil, destacando as fortalezas e as lacunas dessa produção.

\section{MÉTODO}

Pesquisa do tipo exploratório-descritiva, em base documental, de natureza quantitativa. A produção científica do estado de São Paulo foi definida a partir da consulta ao Currículo Lattes dos 94 pesquisadores com título de Doutor pertencentes a 12 Grupos de Pesquisa de Educação em Enfermagem cadastrados no Censo 2008 do $\mathrm{CNPq} /$ Brasil. A busca e organização dos dados ocorreram no mês de janeiro de 2011 e incluiu publicações do período de 2004 a 2010. O critério para seleção dos currículos dos doutores foi ter a última atualização realizada nos meses de novembro ou dezembro de 2010.

Do total de 832 artigos científicos apresentados no currículo dos pesquisadores, fizeram parte deste estudo os publicados em inglês e/ou português e/ou espanhol com qualificação A1, A2, B1 e B2, conforme Qualis Periódicos/CAPES (2009) para a área de Enfermagem. Justifica-se este recorte por corresponderem aos índices com alto impacto nacional e internacional na produção científica da área e por, atualmente, os artigos serem considerados o meio mais ágil de difusão e socialização do conhecimento científico.

Os artigos científicos foram capturados e organizados por ano em livrarias a partir do gerenciador bibliográfico EndNote ${ }^{\circledR}$. Com o total de artigos selecionados, realizou-se a leitura de todos os resumos e a análise descritiva dos dados. Foram extraídas as seguintes informações dos resumos organizados por ano e numerados sequencialmente em arquivos no Microsoft Word®: identificação (título, autores, periódico), natureza do estudo (qualitativa, quantitativa, quali-quantitativa, relato de experiência, revisão de literatura, reflexão teórica, estudo histórico, ensaio), técnica para coleta de dados, referencial teórico utilizado e tipo de análise dos dados adotada.

Após essa organização inicial, foram definidas sete tendências temáticas, a partir das quais os artigos foram classificados por convergência e aderência a temática, sendo elas: Cuidado em Enfermagem e Saúde; Educação em Enfermagem 
e Saúde; Processo de Trabalho em Saúde; Estudos Epidemiológicos; Atenção à Saúde Mental; Qualidade de Vida; Ética e Bioética; e Outros Temas. Seguindo a lógica apresentada nos estudos ${ }^{5-6}$, na seção Outros Temas foram incluídos temas pontuais que não abordavam o tema de educação e dificilmente estavam relacionados com a linha de pesquisa dos grupos.

Os artigos foram analisados com relação à tradução/aplicação por meio do número de citações expressos em três bases de dados (Web of Science, Scopus e SciELO). Tal quantitativo foi extraído mediante a consulta ao Currículo Lattes dos pesquisadores. Foram consideradas as citações de cada artigo nas diferentes bases de dados de modo cumulativo.

Ainda, foram organizados arquivos referentes aos "periódicos de publicação", nos quais foram expressos: título do artigo, nome do periódico, área principal do periódico e classificação do periódico segundo 0 Qualis PeriódicosEnfermagem/CAPES, ano base 2009. O aplicativo que permite a classificação e consulta ao Qualis das áreas, bem como a divulgação dos critérios utilizados para a classificação de periódicos, é o WebQualis, disponível no portal virtual da CAPES http://qualis.capes.gov.br/webqualis.

Os achados estão apresentados nos resultados por meio de estatística descritiva simples e discutidos com literatura pertinente. Por se tratar de uma pesquisa documental, na qual as informações coletadas são de domínio público, o projeto não foi submetido ao Comitê de Ética em Pesquisa com Seres Humanos. Cumpre destacar que em relação ao tratamento dos dados, os preceitos éticos contidos na Resolução do CNS no 466/12 foram mantidos.

\section{RESULTADOS}

Os Grupos de Pesquisa em Educação em Enfermagem (GPEE) do estado de São Paulo produziram, no período de 2004 a 2010, um total de 557 artigos, distribuídos ao longo do tempo, conforme apresentado na Tabela 1. 
Tabela 1. Artigos científicos dos GPEE de São Paulo, Qualis 2009 - área de Enfermagem CAPES, Brasil, 2011.

\begin{tabular}{ccccccccc}
\hline Qualis/CAPES & $\mathbf{2 0 0 4}$ & $\mathbf{2 0 0 5}$ & $\mathbf{2 0 0 6}$ & $\mathbf{2 0 0 7}$ & $\mathbf{2 0 0 8}$ & $\mathbf{2 0 0 9}$ & $\mathbf{2 0 1 0}$ & Total \\
\hline A1 & 3 & 2 & 2 & - & 4 & 5 & 1 & $\mathbf{1 7}$ \\
A2 & 29 & 32 & 37 & 45 & 40 & 61 & 24 & $\mathbf{2 6 8}$ \\
B1 & 19 & 26 & 20 & 28 & 18 & 32 & 25 & $\mathbf{1 6 8}$ \\
B2 & 13 & 10 & 15 & 12 & 19 & 18 & 17 & $\mathbf{1 0 4}$ \\
Total & $\mathbf{6 4}$ & $\mathbf{7 0}$ & $\mathbf{7 4}$ & $\mathbf{8 5}$ & $\mathbf{8 1}$ & $\mathbf{1 1 6}$ & $\mathbf{6 7}$ & $\mathbf{5 5 7}$ \\
\hline
\end{tabular}

Em relação à natureza dos artigos, constatou-se que 265 correspondem a pesquisas qualitativas, 166 pesquisas quantitativas, 16 estudos quali-quantitativos, 42 correspondem a revisões de literatura, 31 reflexões teóricas, 22 relatos de experiência, 13 estudos históricos e 2 ensaios.

Nos estudos com abordagens qualitativas, as técnicas mais utilizadas para a coleta de dados englobaram: entrevistas semi-estruturadas de caráter individual, observação participante, grupo focal, técnica projetiva (entrevistas abertas e desenhos livres), investigação em situação cotidiana (associação de frases e imagens), escuta ativa, círculos de discussão, grupo operativo. Já para análise dos dados destaca-se a análise temática e análise de conteúdo como predominantes.

Os referenciais teóricos que tiveram maior predominância foram: fenomenologia social com 31 estudos e teoria das representações sociais com 20 estudos. Em menor expressão apresentam-se os referenciais: psicologia social, modelo psicossocial, agir comunicativo, teoria da conscientização de Paulo Freire, interacionismo simbólico e contexto ecológico do desenvolvimento humano.

Referindo-se às pesquisas quantitativas foram expressas as seguintes técnicas de coleta: questionários estruturados e fichas definidas, sendo todos analisados por meio de estatística.

Os estudos de revisões de literatura, estudos históricos e reflexões teóricas foram desenvolvidos a partir da busca de produções científicas em banco de dados, documentos e relatórios oficiais e história oral. 
Tabela 2. Artigos científicos dos GPEE de São Paulo, Qualis Periódicos e área principal de classificação na CAPES, 2009, Brasil, 2011.

\begin{tabular}{|c|c|c|c|}
\hline $\begin{array}{l}\text { Qualis/CAPES- } \\
\text { Enfermagem }\end{array}$ & Periódico & $\mathbf{n}$ & $\begin{array}{l}\text { Área Principal } \\
\text { do Periódico na } \\
\text { CAPES }\end{array}$ \\
\hline \multicolumn{4}{|l|}{ A1* } \\
\hline & Revista de Saúde Pública & 9 & Saúde Coletiva \\
\hline & Midwifery & 2 & Enfermagem \\
\hline & $\begin{array}{c}\text { American Journal of Physiology. Heart } \\
\text { and Circulatory Physiology }\end{array}$ & 2 & Psicologia \\
\hline & Journal of Human Hypertension & 1 & Medicina \\
\hline & $\begin{array}{l}\text { Journal of Wound, Ostomy, and } \\
\text { Continence Nursing }\end{array}$ & 1 & Enfermagem \\
\hline & International Journal of Cardiology & 1 & Medicina \\
\hline & Journal of Clinical Nursing & 1 & Enfermagem \\
\hline
\end{tabular}

A2

\section{Revista Latino-Americana de Enfermagem}

Revista da Escola de Enfermagem da USP

Acta Paulista de Enfermagem

Texto \& Contexto Enfermagem

Cadernos de Saúde Pública

Outros $^{\star *}$

B1
108 Enfermagem

75 Enfermagem

43 Enfermagem

18 Enfermagem

12 Saúde Coletiva

52 Enfermagem

19 Enfermagem

17 Enfermagem

15 Enfermagem

14 Enfermagem

Medicina

Index Enfermería
6

5 Enfermagem 


\section{Qualis/CAPES-}

Enfermagem

Periódico

n do Periódico na

Área Principal

CAPES

Imunologia e

Farmacologia

$4 \quad$ Interdisciplinar Infantil

Outros**

31

B2

\begin{tabular}{|c|c|c|}
\hline Ciência, Cuidado \& Saúde & 22 & Enfermagem \\
\hline Revista Eletrônica de Enfermagem & 21 & Enfermagem \\
\hline Revista Mineira de Enfermagem & 14 & Enfermagem \\
\hline Revista Paulista de Enfermagem & 11 & Enfermagem \\
\hline $\begin{array}{c}\text { Interface - Comunicação, Saúde, } \\
\text { Educação }\end{array}$ & 7 & Interdisciplinar \\
\hline Ciência \& Saúde Coletiva & 6 & Saúde Coletiva \\
\hline Cultura de los Cuidados & 4 & Enfermagem \\
\hline $\begin{array}{l}\text { Rev. da Rede de Enfermagem do } \\
\text { Nordeste }\end{array}$ & 4 & Enfermagem \\
\hline Outros $^{* *}$ & 15 & \\
\hline
\end{tabular}

${ }^{*}$ Visto que o Qualis/CAPES-Enfermagem A1 possui 17 artigos publicados, optou-se por apresentar todos os periódicos, independente de quantitativo de trabalhos/periódico.

**Na categoria "Outros" estão agrupadas as publicações que obtiveram um número inferior a 4 artigos publicados/periódico.

A partir da leitura dos resumos, os estudos foram classificados em 7 tendências temáticas estabelecidas a partir da aderência teórico-conceitual e das similaridades dos conteúdos, conforme expressos na Tabela 3.

Tabela 3. Tendências temáticas dos artigos científicos dos GPEE de São Paulo, Qualis 2009, CAPES, Brasil, 2011.

\begin{tabular}{cccccc}
\hline \multirow{2}{*}{ TENDÊNCIA TEMÁTICA } & \multirow{n}{*}{ Quantitativo Qualis/CAPES } \\
& & A1 & A2 & B1 & B2 \\
\hline Cuidado em Enfermagem e Saúde & 146 & 3 & 62 & 42 & 39
\end{tabular}




\begin{tabular}{cccccc}
\hline \hline \multirow{2}{*}{ TENDÊNCIA TEMÁTICA } & $\mathbf{n}$ & Quantitativo Qualis/CAPES \\
& & A1 & A2 & B1 & B2 \\
\hline Educação, Enfermagem e Saúde & 131 & 2 & 69 & 37 & 23 \\
\hline Estudos Epidemiológicos & 96 & 10 & 46 & 30 & 10 \\
Processo de Trabalho em Saúde & 71 & 1 & 35 & 19 & 16 \\
Atenção à Saúde Mental & 48 & 1 & 23 & 20 & 4 \\
Qualidade de Vida & 17 & - & 6 & 7 & 4 \\
Ética e Bioética & 9 & - & 9 & - & - \\
Outros Temas & 39 & - & 18 & 13 & 8 \\
Total & $\mathbf{5 5 7}$ & $\mathbf{1 7}$ & $\mathbf{2 6 8}$ & $\mathbf{1 6 8}$ & $\mathbf{1 0 4}$ \\
\hline
\end{tabular}

A tendência temática "Cuidado em Enfermagem e Saúde" trouxe como objeto principal dos seus estudos o processo do cuidado, referindo-se a várias dimensões como: saúde da criança e do adolescente, saúde da mulher, saúde do idoso, cuidado domiciliar, cuidado humanizado, acolhimento, cuidado à saúde mental, cuidado de si, cuidado paliativo, processo de morte/morrer.

Os temas mais encontrados na tendência temática "Educação, Enfermagem e Saúde" foram: formação profissional, currículo, tecnologias educacionais, educação permanente em saúde, processo ensino-aprendizagem, práticas pedagógicas, estratégias de ensino, programas educativos, perfil dos estudantes dos programas de pós-graduação, trajetória dos programas de pós-graduação, educação popular.

No que diz respeito à temática "Estudos Epidemiológicos", a maioria dos estudos são farmacológicos, seguido dos estudos sobre doenças cardiovasculares, coeficiente de mortalidade infantil e do idoso, morbi-mortalidade em mulheres e adolescentes, dengue, tuberculose, hanseníase, hipertensão arterial sistêmica, insuficiência renal crônica e acidentes de trabalho.

Já na tendência "Processo de Trabalho em Saúde", constatou-se que os temas mais publicados abordaram: carga de trabalho, sistematização da assistência em enfermagem, riscos ocupacionais, dimensionamento de pessoal, processo de avaliação, cultura organizacional, processo gerencial, perfil assistencial, saúde do trabalhador.

Em relação à temática "Atenção à Saúde Mental", a produção científica concentra estudos sobre: assistência psiquiátrica, reabilitação psicossocial, transtorno 
mental, depressão, uso abusivo de drogas psicoativas, sexualidade do doente mental, Núcleo de Atenção Psicossocial (NAPS), Centro de Apoio Psicossocial (CAPS) e esquizofrenia.

Destaca-se que a temática "Qualidade de Vida" apresenta estudos exclusivos sobre indicadores de qualidade de vida. Já a tendência relativa à "Ética e Bioética" aborda aspectos sobre: ocorrências éticas, comitê de ética, ética gerencial e questões sobre bioética.

Na seção "Outros Temas" encontraram-se estudos sobre: cultura, controle social, questões de gênero, comunicação, história da enfermagem, transplante e doação de órgãos, acupuntura, distúrbios osteomusculares, toxicidade cutânea, medidas de prevenção, inclusão social, homossexualidade.

A Tabela 4 apresenta aspectos relativos ao consumo dos artigos publicados, sendo expresso pelo número de citações dos artigos em bases de dados indexadas.

Tabela 4. Número de citações da produção científica de pesquisadores dos GPEE de São Paulo, citadas em bases de dados indexadas, segundo tendências temáticas, 2011.

\begin{tabular}{|c|c|c|c|c|}
\hline \multirow[b]{2}{*}{ Tendência Temática } & \multirow[b]{2}{*}{$\mathbf{n}$} & \multicolumn{3}{|c|}{ Número de citações } \\
\hline & & SCOPUS & SciELO & $\begin{array}{l}\text { Web of } \\
\text { Science }\end{array}$ \\
\hline Educação, Enfermagem e Saúde & 31 & 53 & 97 & 2 \\
\hline Cuidado em Enfermagem e Saúde & 30 & 71 & 120 & 10 \\
\hline Atenção à Saúde Mental & 24 & 57 & 60 & 9 \\
\hline Estudos Epidemiológicos* & 14 & 56 & 11 & 41 \\
\hline Processo de Trabalho em Saúde & 12 & 21 & 29 & - \\
\hline Outros Temas & 9 & 15 & 14 & 2 \\
\hline Ética e Bioética & 6 & 16 & 15 & - \\
\hline Qualidade de Vida & 1 & 1 & 1 & - \\
\hline Total & 127 & 290 & 347 & 64 \\
\hline
\end{tabular}

\section{DISCUSSÃO}


Os resultados revelam um quantitativo expressivo de artigos científicos (557) publicados pelos GPEE do estado de São Paulo em periódicos com altos índices de impacto, sendo que ao comparar o primeiro e o segundo triênios apresentados na Tabela 1 houve um aumento significativo da produção em 13\%. Ao analisar a natureza dos artigos, percebe-se que a maioria (80\%) são artigos originais de pesquisa. Fato que sugere a adesão por parte dos pesquisadores dos GPEE às políticas de avaliação da CAPES, as quais induzem à busca pelo quantitativo de produção, com predominância de artigos originais, para o fortalecimento das áreas.

O quantitativo expresso nos resultados reforça a hegemonia histórica da Região Sudeste, em especial o estado de São Paulo como "produtor" de conhecimento científico no Brasil, ao menos no que se refere à área da Enfermagem. Ao discutir os dados com estudos publicados sobre o tema de Educação em Enfermagem em outras regiões, em especial a Norte, Nordeste e Centro-oeste, tal quantitativo é de 258 artigos científicos publicados no período 2004-2008, Censo/CNPq 2008 . Desse modo, é possível constatar que, mesmo com políticas indutoras de crescimento da produção científica lançadas pelo Governo Federal na primeira década do Século XXI, ainda há um longo caminho a ser percorrido para se conquistar uma "produção de conhecimento" mais equitativa no Brasil.

Devido ao quantitativo de produção, não há como realizar comparações em relação à produção científica dos GPEE do estado de São Paulo com os demais Estados brasileiros isoladamente. A possibilidade de comparação existente é analisar a produção dos GPEE dos triênios 2005-2007 e 2008-2010 em periódicos com alto fator de impacto na Enfermagem (A1, A2, B1, B2) da Região Sul (424 artigos científicos publicados no período de 2004-2008, Censo CNPq-2006) e dos estados do Rio de Janeiro e de Minas Gerais juntos (249 artigos científicos publicados no período de 2005-2009, Censo CNPq-2008) ${ }^{9-10}$. Dentro da possibilidade de comparação existente, a hegemonia do estado de São Paulo se destaca ao se analisar os artigos publicados em periódicos com os referidos índices de impacto.

Considera-se que, entre outros fatores, os principais responsáveis por essa hegemonia são o desenvolvimento socioeconômico do Estado de São Paulo, o qual promove a indução de Ciência \& Tecnologia, com investimentos independentes aos enviados pelo Governo Federal, a força política das instituições de ensino e, em especial, dos programas de pós-graduação, os quais possibilitam a "retroalimentação" 
das ações, por meio de uma lógica de quem mais produz, mais ganha. Destaca-se, assim, o próprio desenvolvimento histórico da Enfermagem, que ano a ano fortalece e amplia seus programas de Pós-Graduação Stricto Sensu, em parte devido aos motivos anteriormente citados, mas em grande parte pelo trabalho, esforço e dedicação das enfermeiras cientistas que, sob nenhuma hipótese, podem ser desmerecidos.

Um aspecto que não pode ser esquecido é que tais resultados reforçam a responsabilidade que os GPEE do estado de São Paulo têm com as demais regiões do país, principalmente as mais distantes, desenvolvendo estratégias de solidariedade que favoreçam a conquista de melhores níveis de produção científica, principalmente os relacionados ao setor de Educação na área de Enfermagem. Para facilitar essa dinâmica, alguns grupos defendem a solidariedade intelectual entre os pesquisadores e a superação de práticas endogênicas de produção, divulgação e consumo do conhecimento ${ }^{11}$.

A partir dos dados apresentados na Tabela 2, pode-se inferir que os GPEE do estado de São Paulo concentram sua produção em periódicos da área de Enfermagem, aspecto importante para o fortalecimento da área como ciência que produz conhecimento. É possível constatar algumas inserções, principalmente nas áreas de medicina e saúde coletiva, com destaque às publicações em periódicos $A 1$, as quais, a partir da análise do escopo dos periódicos, constata-se que não possuem uma interface com o setor de educação em saúde.

Outro aspecto importante a ser analisado é o fato de que $40,5 \%$ do total de artigos dos GPEE estão publicados em periódicos de Enfermagem pertencentes a instituições de ensino do próprio estado de São Paulo, o que representa um quantitativo significativo concentrado em apenas 3 revistas de enfermagem da própria região com alto índice de impacto (A2).

A predominância de estudos qualitativos (47,5\%), identificados nesse estudo, coaduna-se com os estudos dos GPEE da Região Sul, o qual expõe que $42 \%$ dos estudos da região Sul são de natureza qualitativa. ${ }^{5}$ Acredita-se que 0 fato da Enfermagem realizar, predominantemente, pesquisas de natureza qualitativa, possa dificultar o alcance de publicações em periódicos A1. Ao consultar o WebQualis CAPES, constata-se que a maioria dos periódicos com alto índice de impacto, ou seja, indexados em base de dados altamente reconhecidas, apresentam um escopo que 
aceita majoritariamente estudos de natureza quantitativa, relacionado principalmente à biomedicina. Esse fato restringe o envio de manuscritos relativos à temática de Educação em Saúde em Enfermagem, os quais apresentam estudos cujo foco busca compreender o fenômeno a partir de aspectos que consideram o contexto, a história e a experiência em que sujeitos estão inseridos.

Para a superação desse impasse, faz-se necessário a criação de oportunidades que favoreçam a visibilidade da produção e a socialização da produção dos Grupos de Pesquisa em Educação em Enfermagem. Nesse sentido, iniciativas "geradas" em eventos, tais como os Colóquios Panamericanos de Pesquisa em Enfermagem e as Conferências Iberoamericanas de Educação em Enfermagem, contribuem com a visibilidade de diferentes setores da Enfermagem, entre eles o da Educação, no cenário iberoamericano, a partir da criação de redes de integração e desenvolvimento de projetos multicêntricos entre diversos países ${ }^{1}$.

Essas iniciativas devem receber incentivos, a fim de que não seja uma prática de poucos, mas sim, que possam ser desenvolvidas pela maioria dos Grupos de Pesquisa em Educação em Enfermagem do Brasil. É imprescindível que os grupos desenvolvam projetos de pesquisa com objetivos comuns, unindo os diversos atores envolvidos dentro desse núcleo, através do trabalho em equipe. Dessa forma, é possível produzir conhecimentos com a mesma finalidade, fortalecendo as linhas de pesquisa e consolidando os grupos do setor de Educação na área de Enfermagem.

Comparando as tendências temáticas dos artigos (Tabela 3) e os periódicos nos quais foram publicados (Tabela 2), chama a atenção o fato de que do total de artigos publicados em periódicos com qualificação $A 1,58,8 \%$ pertencem à tendência temática de "Estudos Epidemiológicos". Esses estudos analisam, de forma quantitativa, a frequência e a distribuição dos fenômenos referentes aos processos de saúde/doença. Indicam taxas ou riscos sobre determinado evento, levam em consideração o tempo, lugar e o objeto para realizar as avaliações ${ }^{12}$, logo, estruturam-se no bojo do pensamento filosófico positivista, corrente ainda hegemônica na produção científica.

O fato da predominância de artigos publicados por estes Grupos de Pesquisa em periódicos $\mathrm{A} 1$ pertencer à referida tendência temática é um aspecto importante para análise e reflexão, pois para se atingir os parâmetros de reconhecimento internacional definido pela CAPES e, por conseguinte, lograr publicações em periódicos de alto impacto, há um indício de que os pesquisadores destes Grupos estão 
produzindo estudos sem aderência à linha de pesquisa referente à educação. A existência de uma significativa produção de artigos em outras temáticas além da Educação em Enfermagem deve-se ao fato de que os GPEE analisados apresentam no mínimo uma linha de pesquisa a mais, além da relativa à educação.

A produção científica do estado de São Paulo apresenta $26,2 \%$ dos estudos pautados na tendência temática "Cuidado em Enfermagem e Saúde". Tal tendência, mesmo sendo a predominante nos artigos publicados, ainda se apresenta inferior ao encontrado em outras regiões brasileiras. A Região Sul apresenta $42 \%$ e as Regiões Norte, Nordeste e Centro-Oeste $44 \%$ dos estudos pautados dentro da temática em questão ${ }^{5-6}$.

A tendência temática "Educação, Enfermagem e Saúde" tem destaque dentro do escopo dos artigos analisados, pois mesmo não sendo a maioria, os artigos dessa temática representam $23,5 \%$ das publicações. Esse aspecto é um fator positivo, denotando que os grupos de pesquisa estão produzindo de forma relativamente articulada dentro da área de Educação em Enfermagem. Os GPEE de São Paulo têm, em índices percentuais, uma produção na temática de educação em enfermagem semelhante ao apresentado em outras regiões do país. Na Região Sul, a referida temática corresponde a $20 \%$ dos artigos analisados; na Região Norte, Nordeste e Centro-Oeste expressam $24 \%$ dos artigos publicados pelos grupos ${ }^{5-6}$.

Outro ponto que merece destaque é a tendência definida como "Outros temas", pois a produção científica classificada nessa tendência mostra uma gama de estudos pontuais que são desenvolvidos pelos GPEE. Isso acarreta em uma dispersão no processo de produção do conhecimento, fragilizando a linha de Educação em Enfermagem.

Com relação à tradução/aplicação do conhecimento produzido, algumas bases de dados criaram mecanismos que possibilitam aferir o quantitativo que determinado artigo foi citado por outros autores. Para o período em que os dados foram coletados, esses quantitativos são considerados válidos, mesmo produzindo uma "análise relativa" sobre a magnitude de citações, visto que indicam apenas os estudos citados dentro de suas próprias bases, pois não há cruzamento de dados em bases de outros editores, com exceção de quando há consórcio entre alguns editores científicos, os quais são raros. 
A Tabela 4 apresenta como os artigos produzidos nos GPEE estão sendo consumidos, segundo citações apresentadas nas bases de dados Scopus, SciELO e Web of Science. Observa-se que, mesmo havendo, nas três bases de dados, um considerável número de citações de artigos referentes às tendências temáticas "Cuidado em Enfermagem e Saúde" e "Educação, Enfermagem e Saúde", destaca-se que o maior número de citações (41) na base considerada mais importante para área da saúde (Web of Science), a qual segue a lógica biologicista de construção de conhecimento, refere-se a apenas 14 artigos da tendência temática de estudos epidemiológicos. Esse fato desperta a importância dos GPEE refletirem sobre que enfoques estão construindo os conhecimentos na área de Educação em Enfermagem, bem como a partir de que produções os pesquisadores "considerados como de Grupos de Pesquisa em Educação em Enfermagem" estão sendo reconhecidos.

Os resultados expõem que em todas as tendências temáticas, entre elas a de "Educação, Enfermagem e Saúde", o maior quantitativo de citações está expresso na base SciELO. Essa base de dados mostra-se para a área da Enfermagem como um importante veículo de divulgação de conhecimento científico, visto que segue uma lógica de acesso livre ao conhecimento - via editor "Biblioteca Virtual da Saúde" (BVS), e apresenta em seu escopo o reconhecimento de que produtos derivados de pesquisas de natureza qualitativa são tão importantes quanto os de natureza quantitativa para o desenvolvimento científico das áreas. Tais aspectos devem ser enaltecidos e valorizados na Enfermagem, pois, como pode ser constatado nos resultados do presente estudo, a natureza qualitativa em pesquisas é a mais utilizada em trabalhos realizados por pesquisadores de Educação em Enfermagem.

\section{CONCLUSÃO}

A produção científica dos Grupos de Pesquisa de Educação em Enfermagem do estado de São Paulo mostra que os mesmos possuem um quantitativo considerável de estudos que estruturam a tendência temática "Educação, Enfermagem e Saúde", porém a temática sobre "Cuidado em Enfermagem e Saúde" ainda se configura com maior expressão. É importante atentar para o quantitativo relativo às demais temáticas, pois expõem uma inclinação que os Grupos de Pesquisa possuem em não conseguir agregar os aspectos relacionados à Educação em 
Enfermagem e Saúde no momento em que trabalham outros temas, evidenciando que os grupos ainda publicam temas variados, muitas vezes fugindo do seu foco principal. Esse fato precisa ser discutido e analisado no interior dos Grupos de Pesquisa de Educação em Enfermagem do estado de São Paulo, com especial atenção às publicações conquistadas em periódicos qualificados como $\mathrm{A} 1$.

Assim, ficam os questionamentos: as produções publicadas por estes Grupos de Pesquisa em periódicos de mais alto impacto na área de Enfermagem (A1) estão efetivamente fortalecendo o setor de Educação da área, com o qual, eticamente, os mesmos deveriam estar comprometidos? Os pesquisadores estão se "curvando" a uma política, por vezes perversa, que estimula a produção por produção, sem considerar efetivamente os movimentos de mudança e o impacto que os processos de construção de conhecimento produzem em um setor para as pessoas/população envolvidas? O que os Grupos de Pesquisa devem efetivamente buscar? Pois, teoricamente, espera-se que os Grupos de Pesquisa em Educação em Enfermagem desenvolvam trabalhos em torno de suas temáticas de interesse ou linhas de pesquisa declaradas.

\section{REFERÊNCIAS}

1. Malvarez SM, Castrillón-Agudelo MC. Panorama de la fuerza de trabajo en enfermería en América Latina. Rev Enferm IMSS. 2006;14(3):145-65.

2. Gomes DC, Prado ML, Canever BP, Jesus BH, Sebold LF, Backes VMS. Doctor of nursing: capacity for building a professional and scientific career Project. Texto Contexto Enferm. 2016;25(3):e1260015. Disponível em: http://www.scielo.br/pdf/tce/v25n3/0104-0707-tce-25-03-1260015.pdf. Acesso: 15 out. 2016.

3. Scochi CGS, Munari DB. A Pós-graduação em enfermagem brasileira faz quarenta anos: avanços, desafios e necessidade de novos empreendimentos. Esc Anna Nery (impr.) 2012;16(2):215-8.

4. Backes VMS, Canever BP, Ferraz F, Lino MM, Prado ML, Reibnitz KS. Grupos de pesquisa de educação em enfermagem da Região Sul do Brasil. Rev Gaúcha Enferm. 2009;30(2):249-56.

5. Lino MM, Backes VMS, Ferraz F, Reibnitz KS, Martini JG. Análise da produção científica dos grupos de pesquisa em Educação em Enfermagem da Região Sul do Brasil. Texto Contexto Enferm. 2010;19(2):265-73. 
6. Schveitzer MC, Backes VMS, Cutolo LEA, Viana LO. Estilos de pensamento em educação em enfermagem: a produção científica de três regiões do Brasil. Escola Anna Nery 2013;17(1):60-7.

7. Prado ML, Backes VMS, Bruggemann OM. Democratização de produção do conhecimento e acessibilidade à informação: desafios para a qualificação da prática de Enfermagem. Biblioteca Lascasas 2008;4(1):3-8.

8. Schveitzer MC, Backes VMS, Lino MM, Canever BP, Gomes DC. Grupos de pesquisa em educação em enfermagem: caracterização de três regiões brasileiras. Texto Contexto Enferm. 2011;20(n.spe):117-23.

9. Lino MM, Backes VMS, Canever BP, Ferraz F, Prado ML. Profile of scientific and technological production in nursing education research groups in the south of Brazil. Rev. Latino-Am. Enfermagem. 2010;18(3):452-8.

10. Gomes DC, Backes VMS, Lino MM, Canever BP, Ferraz F, Schveitzer MC. Produção científica em Educação em Enfermagem: grupos de pesquisa Rio de Janeiro e Minas Gerais. Rev Gaúcha Enferm. 2011;32(2):330-7.

11. Cabral IE, Tyrrel MAR. Pesquisa em enfermagem nas Américas. Rev. Bras Enferm. 2010;63(1):104-10.

12. Rouquayrol MZ, Gurgel M. Epidemiologia e Saúde. 7 ed. Rio de Janeiro: Medbook; 2013. 